\title{
Dynamical analysis and consistent numerics for a delay model of viral infection in phytoplankton population
}

\author{
A. S. Hassan ${ }^{1}$ \\ Department of Mathematical Sciences, Faculty of Physical Sciences, Bayero University, \\ P.M.B. 3011, Kano, Nigeria. \\ Department of Mathematics and Applied Mathematics, University of Pretoria, 002, South \\ Africa.
}

\begin{abstract}
In this article, the effects of time delay and carrying capacity in the dynamics of viral phytoplankton and consistence numerics are studied. Basic properties and stabilities of equilibria are rigorously analyzed and conditions for stability switches are found. A dynamically consistent nonstandard finite difference scheme for the continuous delay model is designed to verify the results and reveal some interesting features of the model. Some ecological implications and interpretations are provided.
\end{abstract}

Keywords: Phytoplankton, Delay, Stability switches, Nonstandard finite difference scheme.

\section{Introduction}

Phytoplankton are microscopic unicellular plants that inhabit the upper sunstruck layer of almost all oceans and bodies of fresh water. Phytoplankton forms the pyramidal base as source of food and energy to both aquatic and terrestrial organisms. On the other hand, they act as environmental stabilizers by consuming the carbon dioxide released in respiration and other chemical processes and subsequently release oxygen which is vital for all living organisms. Under favourable conditions, the phytoplankton biomass can

\footnotetext{
${ }^{1}$ Corresponding author Email: hashitu.mth@buk.edu.ng
} 
grow explosively and followed by rapid decrease periodically - a phenomenon called "bloom" [33, 34]. Amongst the major causative agents of phytoplankton biomass depletion are sinking and grazing by the animal counterparts (zooplankton) in form of predation. However, over the last decade, some laboratory investigations have shown the presence of pathogenic virus attacks as lytic infection in phytoplankton and other marine organisms $[6,14]$. For instance in [14], it has been observed that the addition of viral concentrates reduce the respiration rate of the natural microplankton community by 50 to $78 \%$ and increased the respiration rate of backterioplankton by $92 \%$ on average. According to [6], the viral lysis accounted for between 5 to $66 \%$ of the total mortality of Phaeocystis . However, in real epidemiological context, the infected phytoplankton stay for sometime lag (incubation period) for the virus to mature before becoming fully infective. Along this line, several biological experiments have been conducted to confirm the presence of incubation period in phytoplankton organisms. For example in [14], it has been reported that the incubation period lasted for 24hours at room temperature. Similarly, in [6], using adapted dilution method, 24hours incubation period was used.

From the modeling side, time delay has been incorporated into some mathematical models to represent incubation period, maturation time or wanning time of immunity in biology and epidemiology, see for example [10, 25, 26, 31, 32, 37], and the references there in. In particular, delay phytoplankton and/or zooplankton models were formulated and analyzed. For instance, Dhar and Sharma in [11], observed that phytoplankton species, in the absence of incubated class are unstable around an endemic equilibrium but the presence of incubated class has made it conditionally stable. Thereafter, $\mathrm{Xu}$ in [37], investigated the system in [11], with delay in form of re-growth time for the susceptible phytoplankton population. Using the delay as a bifurcating parameter, $\mathrm{Xu}$ observed that a family of periodic solutions bifurcate from the equilibrium when it exceeds a critical value. On the other hand, in [31], the interaction of toxic phytoplankton-zooplankton organisms has been presented. Using delays to represent times for conversion of nutrients to species and maturity for phytoplankton, they found the parameter range of the delay for hopf bifurcation to occur in addition to the global asymptotic stability for a boundary equilibrium.

The continuous epidemiological models are usually formulated using systems of nonlinear differential equations, whose exact solutions, if at all exists, are very difficult to determined. Furthermore, for delay systems, as noted in [5, 15], finding effective numerical solution is quite challenging. Moreover, in many biological processes, the system is expressed in discrete data such as minutes, days, weeks e.t.c., for easy observation and reporting. Therefore the adaptive numerical schemes such as ODE45, DDE23 e.t.c. are not appropriate for modeling these systems. This necessitate the use of numerical methods, preferably, those that best approximate and replicates the basic properties of these systems in equally spaced time periods. Moreover, as stated in $[13,17,30]$, 
some numerical methods such as Euler, Runge-Kutta, DDE23 etc, fails to properly approximate the solutions of the continuous model, producing spurious, chaos and negative solutions, among other reasons, due to the step sizes used in the simulations. To support some of these claims, in [36] although positivity of solutions at all times was proved, however, negative values were observed in the solution profiles. Based on these facts, we present the nonstandard finite difference (NSFD) scheme, base on the frame work proposed by Mickens [28, 29] and elaborated further by [1, 3], which is shown to be dynamically consistent with the basic dynamics of the continuous model, regardless of the step size use. The delay term is realistically discretized using the theta - method as proposed in [16]. The known discrete stability methods, such as Jury and Schur-Cohn criteria are realistically too challenging to implement. As such, we use the numerical simulations to prove the stabilities of the fixed points.

This article is therefore of twofold. In the first part, we extend the study in [11] by incorporating delay as an incubation period of the viral phytoplankton to form a system of delay differential equations. The objective is to see the effects of time delay and other parameters in the dynamics of the model. In the second part, a reliable NSFD scheme which replicates the realistic dynamical behaviour of the delay model, is designed and analyzed. The basic properties of the continuous delay model are rigourously studied, amongst which, three equilibria points are obtained. The trivial and the disease free equilibria are unconditionally inside the biological feasible region, while the endemic (disease) equilibrium, under certain condition on a threshold quantity. Furthermore, the asymptotic stability analyses are rigorously investigated. In particular, the phenomenon of stability switches (Hopf bifurcation), with carrying capacity and delay as bifurcation parameters, are established. Ecologically, the conditions for eradicating the viral attack and co-existence between the viral and susceptible phytoplankton are given. At this juncture, it is worth stating that, in addition to testifying the results in [11], our findings also reveals the phenomenon of stability switches and global stabilities of the equilibria in addition to showing the effect of delay on the infectivity.

The rest of the paper is organized as follows. In Section 2, we present the details for the construction of the model, the basic properties of the model, the stabilities of equilibria and threshold analysis for the effect of delay on the viral infection. In Section 3 , we consider the dynamics of the NSFD scheme. While numerical simulations are presented in Section 4 followed by concluding remarks. 


\section{The model}

The model is presented by the following system of delay differential equations:

$$
\begin{aligned}
& \frac{d P_{s}(t)}{d t}=r P_{s}(t)\left(1-\frac{P_{s}(t)}{K}\right)-\alpha P_{s}(t) P_{i}(t-\tau) e^{-\beta \tau}+\gamma P_{i}(t), \\
& \frac{d P_{i}(t)}{d t}=\alpha P_{s}(t) P_{i}(t-\tau) e^{-\beta \tau}-\beta P_{i}(t) .
\end{aligned}
$$

Here, $P_{s}(t)$ and $P_{i}(t)$ are the population densities of susceptible and viral (infected) phytoplankton respectively, at time $t$. The parameter $r$ is the intrinsic growth rate of the population of susceptible phytoplankton, which is assumed to grow logistically with carrying capacity $K$. The parameter $\alpha$ is the disease contact rate and $\beta$ the removal rate of the diseased phytoplankton and also natural death rate, out of which $\gamma$ is the fraction of infected phytoplankton recovered dead mass that rejoin the susceptible phytoplankton population in form of nutrients uptake. The variable $P_{i}(t-\tau)$, is the population of phytoplankton that were infected at $(t-\tau)$ time ago but will only infect a susceptible phytoplankton after elapse of $\tau$ time. Here $\tau$ is the incubation period of the viral phytoplankton. The term $e^{-\beta \tau}$, is proportional to the probability that an infected phytoplankton survives natural death $\beta$ during the time period $\tau$, to spread the disease. It is assumed that the removal rate $\beta$, is greater than $\gamma$. The total population at time $t$ is given by

$$
P_{t}(t)=P_{s}(t)+P_{i}(t)
$$

The model (1) satisfy the nonnegative initial conditions given by

$$
P_{s}(t)=\phi_{1}(t), P_{i}(t)=\phi_{2}(t), t \in[-\tau, 0],
$$

where $\phi_{1}, \phi_{2}$, are continuous functions mapping the interval $[-\tau, 0]$ into $\mathbb{R}_{+}^{2}$.

\subsection{Basic properties of the model}

The basic properties of the model, which include existence, uniqueness, boundedness, positivity, stabilities of equilibria can now be studied.

To prove the existence, uniqueness and positivity of solution, we claim the following result:

Theorem 1. The solution $\left(P_{s}(t), P_{i}(t)\right)$ of the model (1) with initial conditions (3), exists for all $t \geq 0$. In addition, $P_{s}(t) \geq 0, P_{i}(t) \geq 0$ for all time, $t>0$.

Proof. For existence and uniqueness of solution, the system (1), can be expressed as

$$
\frac{d X}{d t}=f\left(t, X_{t}\right)
$$


where $f$ is Lipschitz continuous function while $X(t)=\left(P_{s}(t), P_{i}(t)\right.$. It follows from Theorem 2.2 in [21] that the system (1) has a unique solution $\left(P_{s}(t), P_{i}(t)\right)$ whenever (3) is satisfied.

The positivity of solution can be proved by the method of contradiction as follows: Assume that $P_{s}(t) \geq 0$ and $P_{i}(t) \geq 0$ for all $t \in[-\tau, 0]$ and that $P_{s}(t)<0, P_{i}(t)<0$ for $t \in(0, \infty)$. Then by the continuities of $P_{s}(t), P_{i}(t)$ there exists times $t^{0} \in[0, \infty)$, $t^{1} \in[0, \infty)$ where signs changes at least once, such that $P_{s}(t)>0, P_{i}(t)>0$, for any $0<t<t^{0}, 0<t<t^{1}, P_{s}\left(t^{0}\right)=0$ and $\frac{d P_{s}\left(t^{0}\right)}{d t} \leq 0$. Without loss of generality, assume that $t^{0}<t^{1}$.

Consider the first equation of (1),

$$
\begin{aligned}
\frac{d P_{s}\left(t^{0}\right)}{d t} & =r P_{s}\left(t^{0}\right)\left(1-\frac{P_{s}(t)}{K}\right)-\alpha P_{s}\left(t^{0}\right) P_{i}\left(t^{0}-\tau\right) e^{-\beta \tau}+\gamma P_{i}\left(t^{0}\right) \\
& =\gamma P_{i}\left(t^{0}\right) \\
& >0
\end{aligned}
$$

which contradicts the earlier assumption that $\frac{d P_{s}}{d t}\left(t^{0}\right) \leq 0$. Hence there is no such time $t^{0}$. Therefore, $P_{s}(t) \geq 0$ at all time $t>0$.

Using the same argument, suppose that $P_{i}\left(t^{1}\right)=0$, and $\frac{d P_{i}(t)}{d t} \leq 0$. Then It follows from the second equation in (1), that

$$
\begin{aligned}
\frac{d P_{i}\left(t^{1}\right)}{d t} & =\alpha P_{s}\left(t^{1}\right) P_{i}\left(t^{!}-\tau\right) e^{-\beta \tau}-\beta P_{i}\left(t^{1}\right), \\
& =\alpha P_{s}\left(t^{1}\right) P_{i}\left(t^{1}-\tau\right) e^{-\beta \tau}, \\
& >0
\end{aligned}
$$

again, contradicting the assumption that $\frac{d P_{i}(t)}{d t} \leq 0$. Therefore $P_{i}(t) \geq 0$ for all $t>0$. Hence the solution $\left(P_{s}(t), P_{i}(t)\right)$ is nonnegative for all time $t>0$. This proves the Theorem.

\subsubsection{Invariant region}

Theorem 2. The biologically-feasible region, defined as $\Gamma=\left\{\left(P_{s}, P_{i}\right) \in \mathbb{R}_{+}^{2}: P_{s}+P_{i} \leq \frac{K r}{\eta}\right\}$, where $\eta=\min (\beta-\gamma, r)$, is positively-invariant.

Proof. Adding the two equations in (1), we have:

$$
\frac{d P_{t}}{d t}=r P_{s}(t)\left(1-\frac{P_{s}(t)}{K}\right)-(\beta-\gamma) P_{i}(t)
$$


Let $\eta$ be the minimum of $(\beta-\gamma, r)$, then

$$
\frac{d P_{t}}{d t}+\eta P_{t}(t) \leq r P_{s}(t)\left(2-\frac{P_{s}(t)}{K}\right)=g\left(P_{s}\right)
$$

where, the function $g$ is maximum at $P_{s}=K$, therefore, we can let $g\left(P_{s}\right) \leq K r$.

$$
\begin{aligned}
\frac{d P_{t}}{d t}+\eta P_{t}(t) & \leq K r, \text { or } \\
\frac{d P_{t}}{d t} & \leq K r-\eta P_{t}(t) .
\end{aligned}
$$

It follows from the Gronwall lemma [20] that

$$
P_{t}(t) \leq P_{t}(0) e^{-\eta t}-\frac{K r}{\eta}\left(e^{-\eta t}-1\right)
$$

In particular,

$$
P_{t}(t) \leq \frac{K r}{\eta}, \text { if } P_{t}(0) \leq \frac{K r}{\eta}
$$

Thus $P_{t}(t)$ is bounded and all solutions with positive initial conditions, starting in the region $\Gamma$, will approach, stay or enter the region, as $t$ tends to $\infty$.

\subsubsection{Existence of equilibria}

At the steady state, in the absence of viral infection, the disease free equilibria of the model (1), obtained by setting the right hand side of system (1) to zero, are given by:

$$
\begin{aligned}
\mathcal{E}_{0}=\left(P_{s}^{*}, P_{i}^{*}\right) & =(0,0), \\
\mathcal{E}_{1}=\left(P_{s}^{* *}, P_{i}^{* *}\right) & =(K, 0) .
\end{aligned}
$$

While in the presence of viral infection, the endemic equilibrium (EE), is obtained to be:

$$
\mathcal{E}_{2}=\left(P_{s}^{* * *}, P_{i}^{* * *}\right)=\left(\frac{\beta e^{\beta \tau}}{\alpha}, \frac{r \beta e^{\beta \tau}}{\alpha(\beta-\gamma)}\left(1-\frac{\beta e^{\beta \tau}}{K \alpha}\right)\right)
$$

It can be seen that the disease free equilibria are unconditionally in $\Gamma$, while $\mathrm{EE}$, with the assumption $(\beta>\gamma)$, is in $\Gamma$ whenever $K>\frac{\beta e^{\beta \tau}}{\alpha}$. 


\section{$2.2 \quad$ Stability analysis}

To determine the local asymptotic stability of the equilibria, we linearize the system (1) about the equilibria, to have

$$
\frac{d \mathbf{Z}}{d t}=J_{1} \mathbf{Z}(t)+J_{2} \mathbf{Z}(t-r)
$$

where $\mathbf{Z}$, is a vector with components $Z_{i j}(t)=\left(P_{s}(t), P_{i}(t)\right)^{T}$ and $J_{1}=\left(a_{i j}\right), J_{2}=\left(b_{i j}\right)$, for $i, j=1,2$ are the Jacobian matrices of (1) given below.

$$
J_{1}=\left[\begin{array}{cc}
r\left(1-\frac{2 P_{s}}{K}\right)-\alpha P_{i} e^{-\beta \tau} & \gamma \\
\alpha P_{i} e^{-\beta \tau} & -\beta
\end{array}\right] \quad \text { and } \quad J_{2}=\left[\begin{array}{cc}
0 & -\alpha P_{i} e^{-\beta \tau} \\
0 & \alpha P_{i} e^{-\beta \tau}
\end{array}\right]
$$

\subsubsection{Stability of disease free equilibria}

Theorem 3. The trivial equilibrium $\mathcal{E}_{0}$ is unstable. However, the disease free equilibrium $\mathcal{E}_{1}$ is absolutely stable when $K<\frac{\beta e^{\beta \tau}}{\alpha}$.

Proof. Assuming a solution of the form

$$
\mathbf{Z}(t)=\mathbf{c} e^{\lambda t},
$$

where $\mathbf{c}$ is a constant vector and $\lambda$ a complex eigenvalue. At $\mathcal{E}_{0}$, equation (7), gives the characteristic equation

$$
H_{0}(\lambda)=(\lambda-r)(\lambda+\beta)=0 .
$$

Hence, the eigenvalues are $r$ and $-\beta$. Thus, the trivial equilibrium $\mathcal{E}_{0}$, is a saddle point, therefore unstable.

For the stability of $\mathcal{E}_{1}$, substituting the nontrivial solution of (8) in equation (7) we have the transcendental equation

$$
H_{1}(\lambda)=(\lambda+r)\left(\lambda+\beta-\alpha K e^{-\tau(\lambda+\beta)}\right)=0 .
$$

It can be seen that one of the eigenvalues of $H_{1}, \lambda=-r<0$. We therefore consider the stability test on the remaining equation

$$
\lambda+\beta-\alpha K e^{-\tau(\lambda+\beta)}=0
$$

as follows. When $\tau=0$, equation (11) gives $\lambda+\beta-\alpha K=0$. Therefore (11) is stable when $K<\frac{\beta}{\alpha}<\frac{\beta e^{\beta \tau}}{\alpha}$. Substituting $\lambda=i y$ (for any $y \in \mathbb{R}_{+}$), in (11)and simplifying, we obtain $F_{1}(y)=y^{2}+\beta^{2}-\alpha^{2} K^{2} e^{-2 \beta \tau}=0$. Hence it can be seen that if $K<\frac{\beta e^{\beta \tau}}{\alpha}, F_{1}(y)$ has no real zeros. This implies that there is no $y \in \mathbb{R}_{+}$that can satisfy (11) Hence, under these conditions, $\mathcal{E}_{1}$ is locally asymptotically stable for all delays $\tau \geq 0$ (absolutely stable). 
Remark 1: The biological interpretation of Theorem 2 is that, when the carrying capacity of the susceptible phytoplankton is less than the threshold value $\left(\frac{\beta e^{\beta \tau}}{\alpha}\right)$, the viral phytoplankton population will face extinction while the susceptible phytoplankton will grow up to the maximum carrying capacity for any value of the delay. Hence the disease will die out in the phytoplankton population.

Furthermore, with $\mathcal{E}_{1}$ as the unique stable equilibrium in $\Gamma$ and to ensure complete eradication of the viral infection irrespective of the initial population started with, we show that it is also globally asymptotically stable in the following result:

Theorem 4. The disease free equilibrium $\mathcal{E}_{1}$ is globally asymptotically stable (GAS) for all delay $\tau \geq 0$ whenever $K<\frac{\beta e^{\beta \tau}}{\alpha}$.

Proof. Consider the model (1) with $K<\frac{\beta e^{\beta \tau}}{\alpha}$ and let $V(t)$ be the Lyapunov function defined by

$$
V(t)=P_{s}(t)-P_{s}^{* *}-P_{s}^{* *} \ln \left(\frac{P_{s}(t)}{P_{s}^{* *}}\right) .
$$

It can be seen that $V(t)$ is non-negative definite in $\Gamma$, with respect to $\mathcal{E}_{1}$. The derivative $\dot{V}$, along the solution of system (1) is given by

$$
\dot{V}=\dot{P}_{s}-\frac{P_{s}^{* *}}{P_{s}} \dot{P}_{s}
$$

Substituting the derivative from system (1) in (13) we have,

$$
\begin{aligned}
\dot{V} & =r P_{s}(t)\left(1-\frac{P_{s}(t)}{K}\right)-\alpha P_{s}(t) P_{i}(t-\tau) e^{-\beta \tau}+\gamma P_{i}(t) \\
& -\frac{P_{s}^{* *}}{P_{s}(t)}\left[r P_{s}(t)\left(1-\frac{P_{s}(t)}{K}\right)-\alpha P_{s}(t) P_{i}(t-\tau) e^{-\beta \tau}+\gamma P_{i}(t)\right] \\
& =-r P_{s}^{* *}\left(1-\frac{P_{s}(t)}{K}\right)\left(1-\frac{P_{s}(t)}{P_{s}^{* *}}\right)-\alpha P_{s}^{* *} P_{i}(t-\tau) e^{-\beta \tau}\left(\frac{P_{s}^{* *}}{P_{s}(t)}-1\right) \\
& -\gamma P_{i}(t)\left(\frac{P_{s}^{* *}}{P_{s}(t)}-1\right), \\
& =-r K\left(1-\frac{P_{s}(t)}{K}\right)^{2}-\alpha K P_{i}(t-\tau) e^{-\beta \tau}\left(\frac{K}{P_{s}(t)}-1\right) \\
& -\gamma P_{i}(t)\left(\frac{K}{P_{s}(t)}-1\right), \\
& \leq 0 .
\end{aligned}
$$

Since in the absence of disease, the susceptible phytoplankton population at time $t$, $P_{s}(t)$, is less than its population at equilibrium point (carrying capacity), where $P_{s}^{* *}=$ $K$. Again, with $\gamma>0$, it follows that $\dot{V}(t) \leq 0$ and $\dot{V}(t)=0$ if and only if $\left(P_{s}^{* *}, P_{i}^{* *}\right)=$ $(K, 0)$ irrespective of the delay value. Therefore, by LaSalle's invariance principle [24], the equilibrium is the largest invariant set in $\Gamma$ such that $\left(P_{s}(t), P_{i}(t)\right) \rightarrow(K, 0)$ as $t \rightarrow \infty$. Thus, $\mathcal{E}_{1}$ is globally asymptotically stable in $\Gamma$ for all delays $\tau \geq 0$. 
Theorem 3 shows that irrespective of the delay value, for any initial conditions, the viral infection of phytoplankton population can be eliminated whenever the carrying capacity of the susceptible phytoplankton population density is less than the threshold value $\left(\frac{\beta e^{\beta \tau}}{\alpha}\right)$.

\subsubsection{Stability of endemic equilibrium}

Similarly, at the endemic equilibrium, $\mathcal{E}_{2}=\left(P_{s}^{* * *}, P_{i}^{* * *}\right)$, as given in (6), we claim the following results:

Theorem 5. The endemic equilibrium $\mathcal{E}_{2}$ is locally asymptotically stable for all delays $\tau \geq 0$ whenever $\frac{2 \beta e^{\beta \tau}}{\alpha}<K<\frac{3 \beta e^{\beta \tau}}{\alpha}$. Furthermore, as $\tau$ increases there will be stability switches for fixed parameter values up to $\tau^{*}$ (critical delay) value and the endemic equilibrium is locally asymptotically stable for $0 \leq \tau<\tau^{*}$ when $K>\frac{3 \beta e^{\beta \tau}}{\alpha}$.

Proof. At the endemic equilibrium, assuming solution of the form in (8), the transcendental polynomial is defined as

$$
H_{2}(\lambda)=P_{2}(\lambda)+Q_{2}(\lambda) e^{-\lambda \tau}=0
$$

with,

$$
\begin{aligned}
P_{2}(\lambda) & =\lambda^{2}+A_{1} \lambda+A_{2}, \\
Q_{2}(\lambda) & =-\beta \lambda+A_{3}
\end{aligned}
$$

where

$$
\begin{aligned}
& A_{1}=\beta\left(1+\frac{r e^{\beta \tau}}{\alpha K}\right)+\frac{r \gamma}{\beta-\gamma}\left(1-\frac{\beta e^{\beta \tau}}{\alpha K}\right), \\
& A_{2}=\frac{r \beta^{2} e^{\beta \tau}}{\alpha K} \\
& A_{3}=r \beta\left(1-\frac{2 \beta e^{\beta \tau}}{\alpha K}\right) .
\end{aligned}
$$

When $\tau=0$, (15) is given by:

$$
H_{2}(\lambda)=\lambda^{2}+\left[\frac{r \beta}{\alpha K}+\frac{r \gamma}{(\beta-\gamma)}\left(1-\frac{\beta}{\alpha K}\right)\right] \lambda+r \beta\left(1-\frac{\beta}{\alpha K}\right) .
$$

It can be seen that the coefficients of $\lambda$ and the constant term are positive if $K>$ $\frac{2 \beta e^{\beta \tau}}{\alpha}>\frac{\beta}{\alpha}$. Therefore, using Discarte's rule of sign, $H_{2}(\lambda)$ has no positive roots and hence is stable when $\tau=0$.

Furthermore, define $F_{2}(\lambda):=\left|P_{2}(\lambda)\right|^{2}-\left|Q_{2}(\lambda)\right|^{2}$. Substituting $\lambda=i y$ in $F_{2}(y)$, for $y \in \mathbb{R}_{+}$, we have,

$$
F_{2}(y)=y^{4}+\left(A_{1}^{2}-2 A_{2}-\beta^{2}\right) y^{2}+\left(A_{2}^{2}-A_{3}^{2}\right), \text { where } A_{2}-A_{3}=-r \beta\left(1-\frac{3 \beta e^{\beta \tau}}{\alpha K}\right) .
$$


From the expressions of the coefficient of $y^{2}$ it can easily be seen that it is positive. While for the constant term $A_{2}^{2}-A_{3}^{2}>0$ if $\frac{2 \beta e^{\beta \tau}}{\alpha}<K<\frac{3 \beta e^{\beta \tau}}{\alpha}$. Therefore, using Discarte's rule of sign, there will be no sign change in $F_{2}(y)$. Hence, $F_{2}(y)$ has no positive root. This implies that $\mathcal{E}_{2}$ is locally asymptotically stable for all delays $\tau \geq 0$ when $\frac{2 \beta e^{\beta \tau}}{\alpha}<K<\frac{3 \beta e^{\beta \tau}}{\alpha}$.

However, if $K>\frac{3 \beta e^{\beta \tau}}{\alpha}$, coefficient of $y^{2}$ is still positive while $A_{2}^{2}-A_{3}^{2}<0$. It follows from Discarte's rule that there is one sign change in $F_{2}(y)$. Therefore, $F_{2}(y)$ has at most a finite number of real zeros (positive and negative).

From above, it is evident that $F_{2}(y)$, with $K>\frac{3 \beta e^{\beta \tau}}{\alpha}$, has one positive root given by:

$$
y=\left[\frac{-B+\sqrt{B^{2}+4 C}}{2}\right]^{1 / 2},
$$

where $B=A_{1}^{2}-2 A_{2}-\beta^{2}, C=\left(A_{2}^{2}-A_{3}^{2}\right)$.

For the root $y$ to be simple, it is suffice to show that at that point, where the stability switch take place, the direction of crossing from left to right of the half-plane and vice vasa, $s \neq 0$, where, $s=\operatorname{sign} F_{2}^{\prime}(y)$.

Hence, $s=F_{2}^{\prime}(y)=4 y^{3}+2\left(A_{1}^{2}-2 A_{2}-\beta^{2}\right) y \neq 0$. Therefore, $y$ is a simple positive root.

Hence, as $\tau$ increase there will be $k \in \mathbb{Z}^{+}$number of stability switches for fixed parameter values and the equilibrium $\mathcal{E}_{2}$ is locally asymptotically stable for $0 \leq \tau<\tau^{*}$.

To determine $\tau^{*}$, we substitute $\lambda=i y$ in (15). Simplifying and separating the real and imaginary parts, we have:

$$
\begin{aligned}
& \sin y \tau=\frac{-P_{2 R} Q_{2 I}+P_{2 I} Q_{2 R}}{\left|Q_{2}\right|^{2}}, \\
& \cos y \tau=-\frac{P_{2 R} Q_{2 R}+P_{2 I} Q_{2 I}}{\left|Q_{2}\right|^{2}},
\end{aligned}
$$

where, $P_{2 R}=A_{2}-y^{2}, \quad P_{2 I}=A_{1} y, \quad Q_{2 R}=A_{3}, \quad Q_{2 I}=-\beta y$.

Therefore, we seek for a unique value of $\theta=y \tau$, such that $\theta \in[0,2 \pi]$ that will satisfy (18). As observed, the signs of the two equations in (18), are positive, hence $\theta$, must satisfy:

$$
\theta=\cot ^{-1}\left(-\frac{P_{2 R} Q_{2 R}+P_{2 I} Q_{2 I}}{-P_{2 R} Q_{2 I}+P_{2 I} Q_{2 R}}\right),
$$

with $0<\theta<\frac{\pi}{2}$. This gives, $\tau^{*}=\frac{\theta}{y}$.

In general, we obtain a sequence of positive values of delay $\tau_{n}$ given by:

$$
\tau_{n}=\frac{(\theta+2 n \pi)}{y}, \text { for } n=0,1,2, \cdots .
$$

Thus, for fixed parameter values, there exists an integer $k$, such that $\tau^{*}=\tau_{0}<\tau_{1}<$ $\tau_{2}<\cdots<\tau_{k-1}<\tau_{k}$, as $\tau$ varies from 0 to $\tau_{k}$, we have alternately, switching from stability, when $0 \leq \tau<\tau_{0}, \tau_{1}<\tau<\tau_{2}, \cdots$, to instability when $\tau_{0}<\tau<\tau_{1}, \tau_{2}<\tau<\tau_{3} \cdots$, 
and back to stability $k$ times, and eventually, unstable for all $\tau>\tau_{k}$.

Remark 2: The biological interpretation of Theorem 5 is that, when the carrying capacity of the susceptible phytoplankton population density is less than $\frac{3 \beta e^{\beta \tau}}{\alpha}$, both susceptible and viral phytoplanktons coexists and the disease will persist for any value of the incubation period. Furthermore, when the carrying capacity of the susceptible phytoplankton population density is greater than the threshold value $\left(\frac{3 \beta e^{\beta \tau}}{\alpha}\right)$, the viral infection will persist in the phytoplankton population with increase in the incubation period up to certain value $\left(\tau^{*}\right)$. Beyond this delay value, there will be fluctuation of phytoplankton populations resulting in periodic solution around the equilibrium $\mathcal{E}_{2}$.

Sequel to analysis in Theorem 5, we can also show that the endemic equilibrium, $\mathcal{E}_{2}$ is globally asymptotically stable when $\frac{2 \beta e^{\beta \tau}}{\alpha}<K<\frac{3 \beta e^{\beta \tau}}{\alpha}$.

Theorem 6. Suppose that $\frac{2 \beta e^{\beta \tau}}{\alpha}<K<\frac{3 \beta e^{\beta \tau}}{\alpha}$, then the interior equilibrium $\mathcal{E}_{2}=$ $\left(P_{s}^{* * *}, P_{i}^{* * *}\right)$ is globally asymptotically stable in $\Gamma$ for any delay $\tau \geq 0$.

Proof. Consider the model (1) and assuming $\frac{2 \beta e^{\beta \tau}}{\alpha}<K<\frac{3 \beta e^{\beta \tau}}{\alpha}$. Let $\mathcal{F}(t)$ be the Lyapunov function defined by

$$
\mathcal{F}(t)=P_{s}(t)-P_{s}^{* * *}-P_{s}^{* * *} \ln \left(\frac{P_{s}(t)}{P_{s}^{* * *}}\right)+P_{i}(t)-P_{i}^{* * *}-P_{i}^{* * *} \ln \left(\frac{P_{i}(t)}{P_{i}^{* * *}}\right),
$$

whose derivative $\dot{\mathcal{F}}$, along the solution of system (1) is given by,

$$
\dot{\mathcal{F}}=\dot{P}_{s}-\frac{P_{s}^{* * *}}{P_{s}} \dot{P}_{s}+\dot{P}_{i}-\frac{P_{i}^{* * *}}{P_{i}} \dot{P}_{i}
$$

Similarly, it can be seen that $\mathcal{F}(t)$ is non-negative definite in $\Gamma$, with respect to $\mathcal{E}_{2}$ and zero only at $\mathcal{E}_{2}$. Substituting the derivatives from (1) in (21), we have

$$
\begin{aligned}
\dot{\mathcal{F}} & =r P_{s}(t)\left(1-\frac{P_{s}(t)}{K}\right)-(\beta-\gamma) P_{i}^{* * *}-r P_{s}^{* * *}\left(1-\frac{P_{s}(t)}{K}\right)+\alpha P_{s}^{* * *} P_{i}(t-\tau) e^{-\beta \tau} \\
& -\gamma \frac{P_{s}^{* * *} P_{i}}{P_{s}(t)}-\alpha \frac{P_{i}^{* * *}}{P_{i}(t)} P_{i}(t-\tau) e^{-\beta \tau}+\beta P_{i}^{* * *} .
\end{aligned}
$$

At endemic equilibrium, we have,

$$
(\beta-\gamma)=r \frac{P_{s}^{* * *}}{P_{i}^{* * *}}\left(1-\frac{P_{s}^{* * *}}{K}\right), \alpha=\frac{\beta e^{\beta \tau}}{P_{s}^{* * *}} \text { and } P_{i}(t-\tau)=P_{i}(t)=P_{i}^{* * *}
$$


Substituting (23) in (22) and simplifying,

$$
\begin{aligned}
\dot{\mathcal{F}} & =r P_{s}(t)\left(1-\frac{P_{s}(t)}{K}\right)-\frac{r P_{i}(t) P_{s}^{* * *}}{P_{i}^{* * *}}\left(1-\frac{P_{s}^{* * *}}{K}\right)-r P_{s}^{* * *}\left(1-\frac{P_{s}(t)}{K}\right) \\
& +\frac{r P_{i}(t) P_{s}^{* * * 2}}{P_{s}(t) P_{i}^{* * *}}\left(1-\frac{P_{s}^{* * *}}{K}\right)+2 \beta P_{i}^{* * *}-\frac{\beta P_{i}(t) P_{s}^{* * *}}{P_{s}(t)}-\frac{\beta P_{i}^{* * * 2} P_{s}(t)}{P_{s}^{* * *} P_{i}(t)} \\
& =-r P_{s}^{* * *}\left(1-\frac{P_{s}(t)}{K}\right)\left[1-\frac{P_{s}(t)}{P_{s}^{* * *}}\right]-\frac{r P_{i}(t) P_{s}^{* * *}}{P_{i}^{* * *}}\left(1-\frac{P_{s}^{* * *}}{K}\right)\left[1-\frac{P_{s}^{* * *}}{P_{s}(t)}\right] \\
& +\beta P_{i}^{* * *}\left[2-\frac{P_{i}(t) P_{s}^{* * *}}{P_{i}^{* * *} P_{s}(t)}-\frac{P_{s}(t) P_{i}^{* * *}}{P_{s}^{* * *} P_{i}(t)}\right], \\
& \leq 0 .
\end{aligned}
$$

Since in the first two terms in (24), at endemic state, susceptible phytoplankton population at $t, P_{s}(t)$, is less than its population at endemic point, $P_{s}^{* * *}$ as a result of viral lysis. While in the third term, the arithmetic mean is greater than or equal to the geometric mean. Hence $2-\frac{P_{i}(t) P_{s}^{* * *}}{P_{i}^{* * *} P_{s}(t)}-\frac{P_{s}(t) P_{i}^{* * *}}{P_{s}^{* * *} P_{i}(t)} \leq 0$.

Therefore with $\frac{2 \beta e^{\beta \tau}}{\alpha}<K<\frac{3 \beta e^{\beta \tau}}{\alpha}$, it follows that $\dot{\mathcal{F}}(t) \leq 0$ and $\dot{\mathcal{F}}(t)=0$ only at $\mathcal{E}_{2}$. Hence, by LaSalle's invariance principle [24], $\left(P_{s}(t), P_{i}(t)\right) \rightarrow\left(P_{s}^{* * *}, P_{i}^{* * *}\right)$ as $t \rightarrow \infty$. Therefore, $\mathcal{E}_{2}$ is globally asymptotically stable in $\Gamma$.

\section{Nonstandard finite difference scheme of the model}

Base on the reasons stated earlier, in this section, we construct a dynamically consistent numerical scheme using the approach of NSFD proposed by Mickens [28]. The NSFD scheme is more capable in replicating the dynamics of the continuous models, irrespective of the step size taken, see for example [1, 2, 17, 29] and some of the references therein. The NSFD scheme of the model is constructed in the following form:

$$
\begin{aligned}
& \frac{P_{s}^{n+1}-P_{s}^{n}}{\psi(\eta, h)}=r P_{s}^{n}\left(1-\frac{P_{s}^{n+1}}{K}\right)-\alpha P_{s}^{n+1} \tilde{P}_{i}^{n} e^{-\beta \tau}+\gamma P_{i}^{n}, \\
& \frac{P_{i}^{n+1}-P_{i}^{n}}{\psi(\eta, h)}=\alpha P_{s}^{n+1} \tilde{P}_{i}^{n} e^{-\beta \tau}-\beta P_{i}^{n},
\end{aligned}
$$

Here and after, $P_{s}^{n} \approx P_{s}\left(t_{n}\right), P_{i}^{n} \approx P_{i}\left(t_{n}\right)$ and $P_{t}^{n} \approx P_{t}\left(t_{n}\right)$, are the approximate numbers of susceptible, infective and total phytoplankton respectively, at time $t_{n}=n h$, while $h$ is the time step size, for $n=0,1,2, \ldots$. The varable $\tilde{P}_{i}^{n}$ is the discretized form of the delay term $P_{i}\left(t_{n}-\tau\right)$, using the theta-method as stated in [16], when $\theta=0$, given as

$$
P_{i}(t-\tau):=\tilde{P}_{i}^{n}=P_{i}\left(t_{n}-\tau\right) \simeq(1-u) P_{i}^{n-m-1}+u P_{i}^{n-m}
$$

Here, $m:=\left[\frac{\tau}{h}\right]$, is the greatest positive integer at most equal to $\frac{\tau}{h}, u=\frac{t_{m+1}-\tau}{\Delta t}$, $0 \leq u \leq 1$. All other parameters have the same meaning and values as in the continuous model (1). 
Using the approach in [29], the denominator function $\psi$ is determined from (25) as follows:

$$
\begin{aligned}
\frac{P_{t}^{n+1}-P_{t}^{n}}{h} & \leq K r-\eta P_{t}^{n+1}, \text { so that } \\
P_{t}^{n+1} & \leq \frac{h\left(K r+P_{t}^{n}\right)}{1+\eta h} .
\end{aligned}
$$

Having $1+\eta h$ appearing in the equation, the step size $h$ could be replaced by a more general denominator function

$$
\psi(\eta, h)=\frac{1-e^{-\eta h}}{\eta}
$$

\subsection{Basic properties of the scheme}

We now show that the NSFD scheme preserves the existence, uniqueness and positivity of solutions at all time as in the continuous model. We claim the following result:

Theorem 7. The NSFD scheme (25) defines a discrete dynamical system in $\Gamma$. Moreover, whenever the initial conditions starting in $\Gamma$ are nonnegative, the scheme generate a unique sequence of positive vectors and $\Gamma$ is an invariant region.

Proof. Assume that the initial conditions $P_{s}(-m-1), P_{i}(-m-1), P_{s}(-m)$, $P_{i}(-m), \ldots, P_{s}(0), P_{i}(0)$ are positive. From $(25)$, solving the variables at the step point $n+1$, we have

$$
\begin{aligned}
P_{s}^{n+1} & =\frac{\left[(1+r \psi) P_{s}^{n}+\gamma P_{i}^{n} \psi\right]}{\left[1+\frac{r P_{s}^{n}}{K} \psi+\alpha \tilde{P}_{i}^{n} e^{-\beta \tau} \psi\right]}, \\
P_{i}^{n+1} & =\frac{\alpha P_{s}^{n+1} \tilde{P}_{i}^{n} e^{-\beta \tau} \psi+P_{i}^{n}(1-\psi \beta)}{1+\psi \beta} .
\end{aligned}
$$

Note that with the choice of the function $\psi$, this gives $\psi \beta<1$. Hence, equations in (29) shows the existence and uniqueness of recursive positive solution sequence $\left(P_{s}^{n+1}\right.$, $P_{i}^{n+1}$ ), whenever we start with positive initial conditions at all times, irrespective of the step size.

For the boundedness of solution in the invariant region, using Lemma 2, we have

$$
\begin{aligned}
\frac{P_{t}^{n+1}-P_{t}^{n}}{h} & \leq K r-\eta P_{t}^{n+1}, \text { so that } \\
P_{t}^{n+1} & \leq \frac{\psi K r+P_{t}^{n}}{1+\psi \eta}, \\
& \leq \frac{K r}{\eta} .
\end{aligned}
$$

This proves the Theorem. 


\subsection{The fixed points of the NFSD scheme}

The fixed points for the NSFD model can be obtained by solving the following system of equations:

$$
\begin{aligned}
& P_{s}^{*}=g\left(P_{s}^{*}, P_{i}^{*}\right) \\
& P_{i}^{*}=j\left(P_{s}^{*}, P_{i}^{*}\right),
\end{aligned}
$$

where $g\left(P_{s}^{*}, P_{i}^{*}\right), j\left(P_{s}^{*}, P_{i}^{*}\right)$ represents the equations at the right hand sides of system (29), respectively. Therefore, at disease free fixed point (DFFP), $P_{i}^{*}=\tilde{P}_{i}^{*}=0$. Substituting this in (31) and simplifying, we have the two disease free fixed points:

$$
\begin{aligned}
& \mathcal{E}_{00}=\left(P_{s}^{*}, P_{i}^{*}\right)=(0,0) \\
& \mathcal{E}_{11}=\left(P_{s}^{* *}, P_{i}^{* *}\right)=(K, 0) .
\end{aligned}
$$

It can be observed that the two disease free fixed points are unconditionally in $\Gamma$ as we have in the continuous model.

In the presence of viral infection, $P_{i}^{* * *} \neq 0$, the endemic fixed point (EFP) can equally be obtained by solving (31). Here, $P_{i}^{*}=\tilde{P}_{i}^{*} \neq 0$. Hence substituting this again in (31), we have the endemic fixed point to be

$$
\mathcal{E}_{22}=\left(P_{s}^{* * *}, P_{i}^{* * *}\right)=\left(\frac{\beta e^{\beta \tau}}{\alpha}, \frac{r \beta e^{\beta \tau}}{\alpha(\beta-\gamma)}\left(1-\frac{\beta e^{\beta \tau}}{K \alpha}\right)\right) .
$$

Similarly, the EFP is in $\Gamma$ whenever $K>\frac{\beta e^{\beta \tau}}{\alpha}$. From the expressions of the fixed points (32) and (33), the NSFD scheme is consistent with the equilibrium points of the continuous model (1).

\subsection{Elementary stabilities of the fixed points}

The local stabilities of the fixed points for the NSFD scheme (25), is achieved whenever all the eigenvalues of the corresponding characteristic polynomial are within a unit circle [19]. The linearized form of (25), can be represented in matrix form as:

$$
Z^{n+1}=Q_{1} Z^{n}+Q_{2} \tilde{Z}^{n}
$$

where, $Z^{n+1}=\left[P_{s}^{n+1}, P_{i}^{n+1}\right]^{T}, Z^{n}=\left[P_{s}^{n}, P_{i}^{n}\right]^{T}$ and $\tilde{Z}^{n}=\left[\tilde{P}_{s}^{n}, \tilde{P}_{i}^{n}\right]^{T}$, representing the delay term, while $Q_{1}$ and $Q_{2}$ are the respective Jacobian matrices given by

$$
Q_{1}=\left[\begin{array}{ll}
q_{1} & q_{2} \\
q_{3} & q_{4}
\end{array}\right] \quad \text { and } \quad Q_{2}=\left[\begin{array}{cc}
0 & q_{5} \\
0 & q_{6}
\end{array}\right]
$$


with $q_{1}=\frac{(1+r \psi)\left(1+\frac{r P_{s} \psi}{K}+\alpha \psi \tilde{P}_{i} e^{-\beta \tau}\right) K-\left((1+r \psi) P_{s}+\gamma P_{i} \psi\right) r \psi}{\left(1+\frac{r P_{s} \psi}{K}+\alpha \psi \tilde{P}_{i} e^{-\beta \tau}\right)^{2} K}, q_{2}=\frac{\gamma \psi}{1+\frac{r P_{s} \psi}{K}+\alpha \psi \tilde{P}_{i} e^{-\beta \tau}}$,

$$
q_{3}=\frac{\alpha \psi \tilde{P}_{i} e^{-\beta \tau}}{1+\beta \psi}, \quad q_{4}=\frac{1}{1+\beta \psi}, \quad q_{5}=-\frac{\left((1+r \psi) P_{s}+\gamma P_{i} \psi\right) \alpha \psi e^{-\beta \tau}}{\left(1+\frac{r P_{s} \psi}{K}+\alpha \psi \tilde{P}_{i} e^{-\beta \tau}\right)^{2}} \text { and } q_{6}=\frac{\alpha \psi P_{s} e^{-\beta \tau}}{1+\beta \psi} .
$$

Here, $P_{s}, P_{i}, \tilde{P}_{i}$ are to be evaluated at the respective fixed points. Since (34) is a linear delay difference equation, we think of a non trivial solution of the form $Z^{n}=\lambda^{n} \mathbf{c}$, where $\mathbf{c}$ is any $2 \times 2$ constant vector. Substituting this in (34) and simplifying, for non trivial solution, we have:

$$
S(\lambda)=\operatorname{det}\left[\lambda \mathbf{I}-Q_{1}-Q_{2}\left[(1-u) \lambda^{-m-1}+u \lambda^{-m}\right]\right]=0,
$$

where $m$ and $u$ have the same meaning as above. By shifting the nodes to the right, (35) can be represented as,

$$
\begin{aligned}
S(\lambda) & =\operatorname{det}\left[\lambda^{m+2} \mathbf{I}-Q_{1} \lambda^{m+1}-Q_{2}[(1-u)+u \lambda]\right]=0, \\
& =\lambda^{2 m+4}-\left(q_{1}+q_{4}\right) \lambda^{2 m+3}+\left(q_{1} q_{4}-q_{2} q_{3}\right) \lambda^{2 m+2}-q_{6} u \lambda^{m+3} \\
& +\left[\left(q_{1} q_{6}-q_{3} q_{5}\right) u-q_{6}(1-u)\right] \lambda^{m+2}+\left(q_{1} q_{6}-q_{3} q_{5}\right)(1-u) \lambda^{m+1}=0 .
\end{aligned}
$$

The stability of the fixed points for the NSFD scheme using the linear delay difference equation (34) is therefore presented by the following theorems:

Theorem 8. The disease free fixed point $\left(P_{s}^{* *}, P_{i}^{* *}\right)$ is locally asymptotically stable for all delays whenever $K<\frac{\beta e^{\beta \tau}}{\alpha}$.

Similarly for the endemic fixed point, we have the following result:

Theorem 9. The endemic fixed point $\left(P_{s}^{* * *}, P_{i}^{* * *}\right)$ is locally asymptotically stable for $0 \leq \tau<\tau^{*}$ if $K>\frac{3 \beta e^{\beta \tau}}{\alpha}$. Furthermore, as $\tau$ increases there will be stability switches.

The results of Theorems 8 and 9 are traditionally verified/proved by using the Jury's conditions [23]. However, this is a challenge, as earlier observed in [5, 15, 27]. Because, for fix $\tau$, the degree $m$ of the polynomial in (36) increases to $\infty$ as $\Delta t$ decreases to zero. Even for the case when $m=0$, the polynomial is of degree 4 (six Jury's conditions to be verified). This make the verification quite demanding, hence we resort to numerical approach (NSFD) to verify these conditions.

\section{Numerical simulations}

In this section, we use the following parameter values from $[11,31]$ to verify the theoretical results using NSFD method.

$$
r=2, \quad \alpha=0.48, \quad \gamma=0.02, \quad \beta=0.16
$$


For the delay $\tau$ and carrying capacity $K$, the values are varied to satisfy the threshold value for stability conditions for each equilibria.

In Figure 1, the dynamical consistency of the NSFD scheme is evident in which the DFFP is shown to be asymptotically stable. In both Figure 1 (a) and (b), different values of the delay $\tau$ and the carrying capacity $K$ are used to show that the fixed point $\mathcal{E}_{11}$ is asymptotically stable in the region $\Gamma$. In Figure 1, different initial data are used

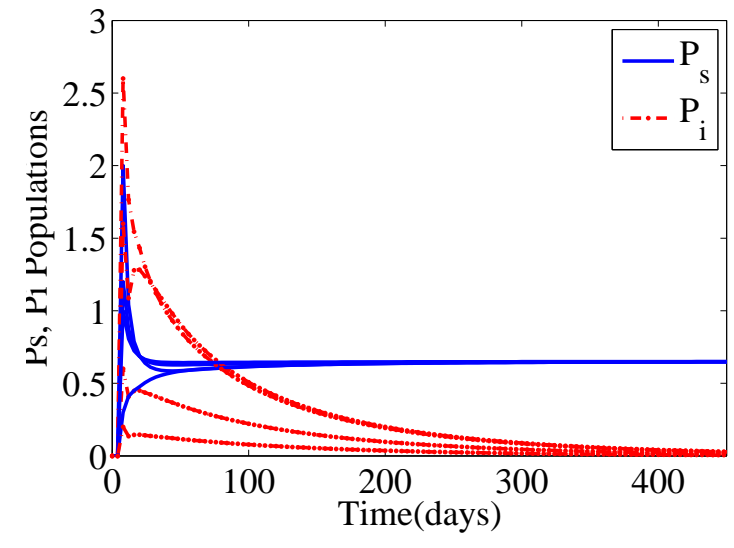

(a)

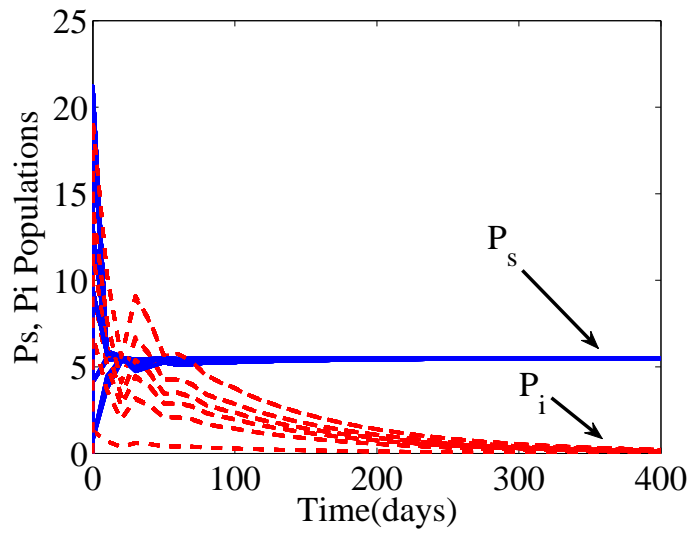

(b)

Figure 1: Numerical simulations using NSFD scheme (29) for solution profiles of phytoplankton populations with different initial functions, showing the GAS of DFE, $\mathcal{E}_{1}$, (a) $\tau=0.2, K=0.282, h=2$, (b) with $\tau=20, K=5.5, h=10$.

to illustrate, using the NSFD scheme (29), the global asymptotic stability of the DFE, $\mathcal{E}_{1}$ (b) for the continuous model (1). It is evident from the two graphs that the scheme preserve the positivity and global stability properties of the continuous model using different delays and step size values.

Figure 2(a) depicts the local asymptotic stability of endemic equilibrium $\mathcal{E}_{2}$ (Theorem 5), using the NSFD scheme (29), with delay $\tau=0.1<\tau^{*}=\tau_{0}=1.6933$. Using different initial data, Figure 2(b) and (c), illustrates the GAS (Theorem 6) of the EE for different delays, $\tau=5$, so that $K=1.95 \in(1.4837,2.2255)$ and $\tau=10$, so that $K=3.95 \in(3.3020,4.9530)$, respectively.

The dynamical consistency of NSFD scheme (25) with the continuous model (1) is portrayed in Figure 3 (a), in which the endemic equilibrium $\mathcal{E}_{2}$ is shown to be unstable when the delay is increased to $\tau=1.8$ beyond the critical delay $\tau^{*}=1.6933$, forming periodic solution around the equilibrium or in (c), stable limit cycle. However, increasing the delay further to $\tau=17.5>\tau_{1}=16.8181$, in (d), the endemic equilibrium $\mathcal{E}_{2}$, is shown to regain stability again. These shows the consistency of the NSFD scheme with regards to the phenomenon of stability switches. Figure 3 (b) display the effect of delay on the infectivity of phytoplankton. Increasing the delay from $\tau=10,13,14,15$, 


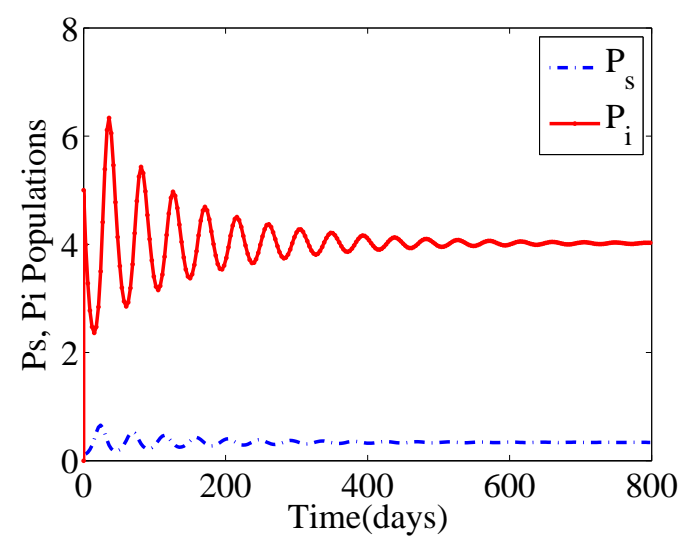

(a)

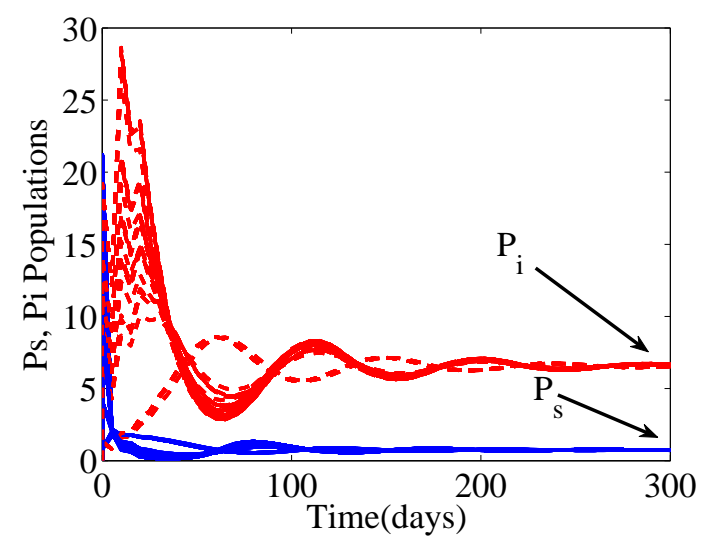

(b)

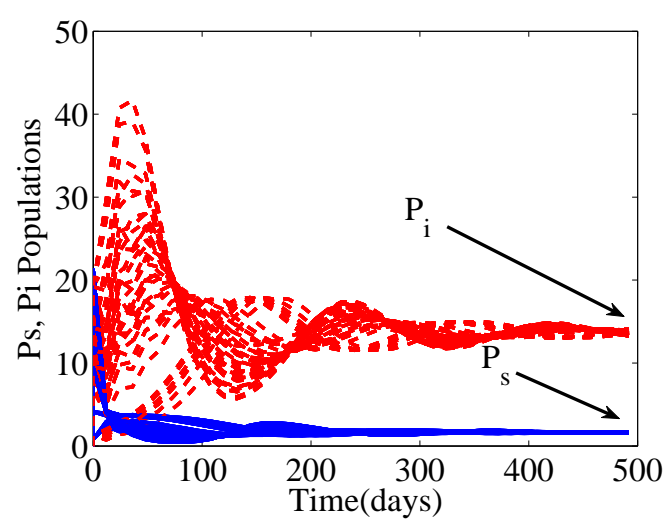

(c)

Figure 2: Numerical simulations using NSFD scheme (29) for solution profiles of phytoplankton populations showing (a) LAS of EE, $\mathcal{E}_{2}, \tau=0.1<\tau^{*}=\tau_{0}=1.6933 \mathrm{~K}=2$, $h=3$, (b) Global asymptotic stability of EE with $\tau=0.5$, so that $K=1.95, h=5$, (c) GAS of $\mathcal{E}_{2}$, with $\tau=10 \in\left(\tau^{*}, \tau_{1}\right)$, so that $K=3.95, h=12$.

the number of infected phytoplankton reduce to $14,12,10,3$ respectively.

Using the same parameter values in (37), we determine the elementary stability of $\mathcal{E}_{11}$ using the NSFD scheme, by verifying the Jury's conditions. In Figure 4 (a) and (b), it can be seen that all the roots $(503,503)$ of the polynomial $(36)$, with degree $m=1000$, lie inside the unit circle for different values of delay. This shows how the NSFD scheme preserves the asymptotic stability of the disease free equilibrium, for all delays, of the continuous model. In Figure 5 (a), it can be seen that all the roots $(503,503)$ of the polynomial $(36)$, with degree $m=1000$, lie inside the unit circle using the same delay and $K$ value as in the continuous model. However, when the delay exceed the critical delay value $\left(\tau=1.8 \in\left(\tau_{0}=1.6933, \tau_{1}\right)\right), \mathcal{E}_{22}$ becomes unstable, which is indicated in Figure 5 (b), with some roots outside the unit circle $\lambda<|1|$. This illustrate how the NSFD scheme preserves the phenomenon of stability switches for the 


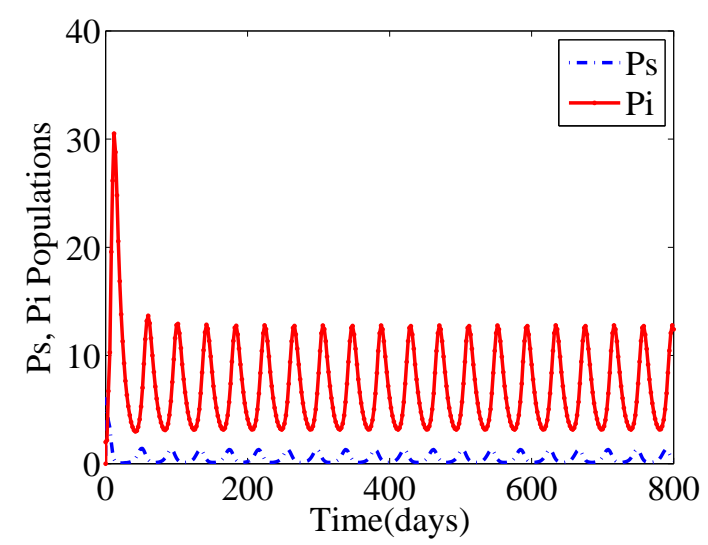

(a)

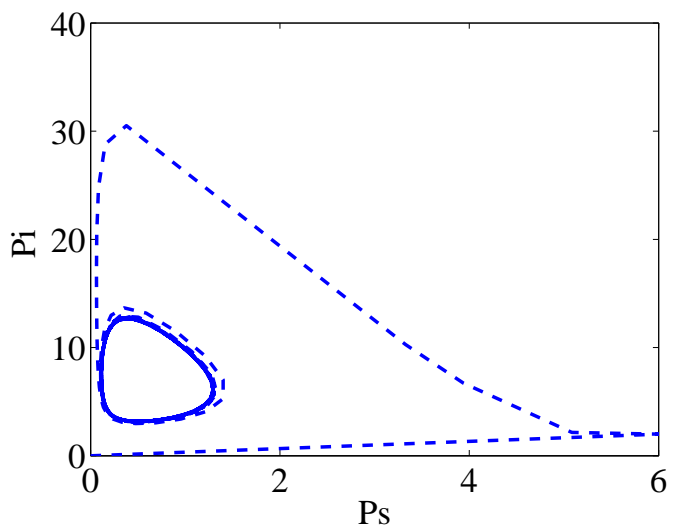

(c)

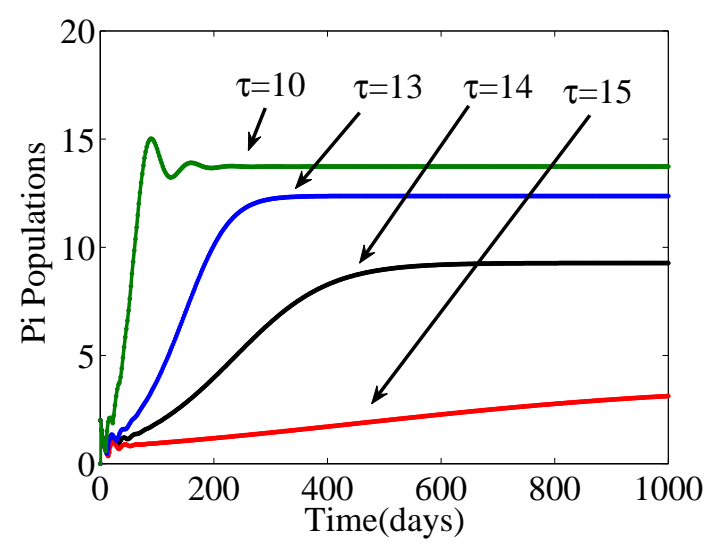

(b)

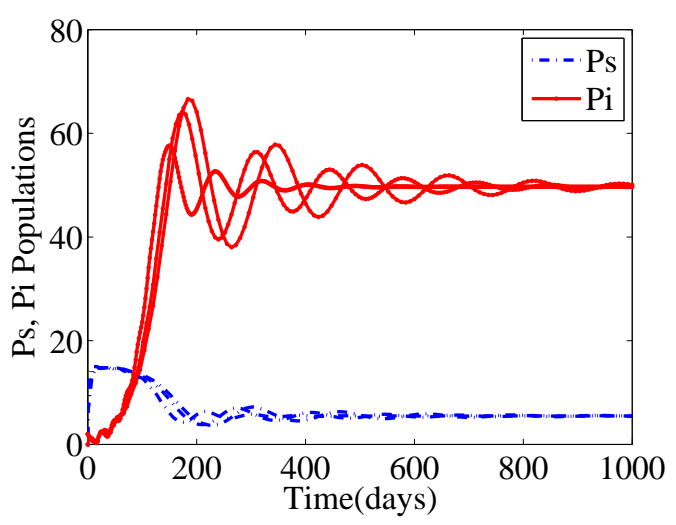

(d)

Figure 3: Numerical simulations using NSFD scheme (29) illustrating (a) oscillations in the profiles of phytoplankton populations around the EE, $\mathcal{E}_{2}$, when $\tau=1.8>\tau^{*}=$ 1.6933, $K=5, h=2$, or in (c) formation of stable limit cycle, parameter values as in (a). in (b), the effect of delay is shown with $K=3.95$ with other parameter values as stated. In (d) Regain of stability for $\mathcal{E}_{2}$, when $\tau=17.5>\tau_{1}=16.8181, K=15$, $h=2,6$ and 8 is shown. 


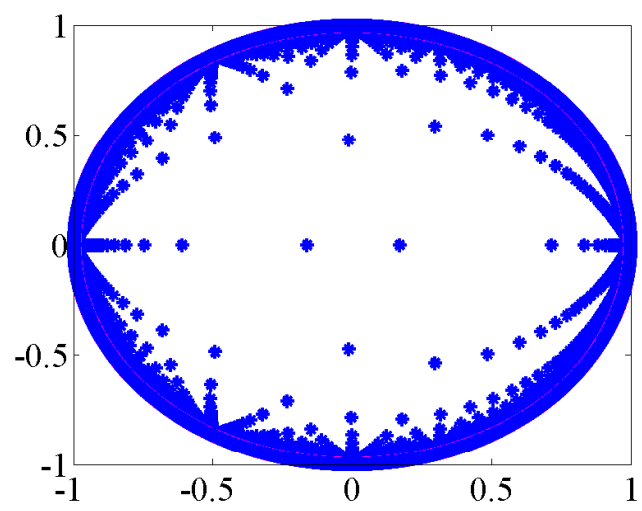

(a)

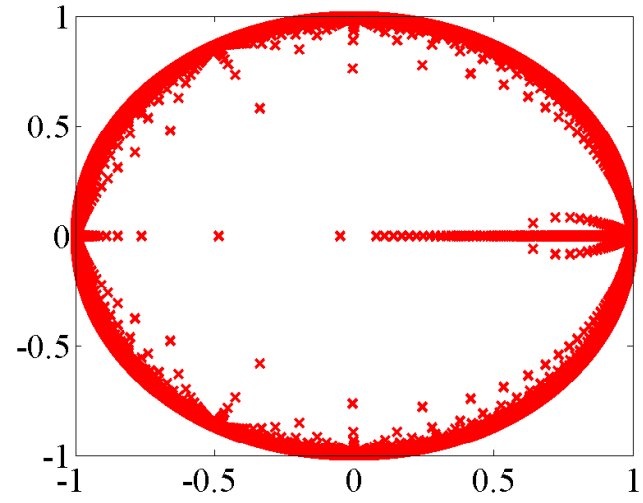

(b)

Figure 4: Numerical simulation showing roots of the polynomial (35) for $m=1000$, within the unit circle indicating the elementary stability of disease free fixed point $\mathcal{E}_{11}$ with (a) $\tau=0.1 \in\left[0, \tau_{0}\right), K=0.282$ (b) $\tau=5 \in\left(\tau_{0}, \tau_{1}\right), K=0.65$

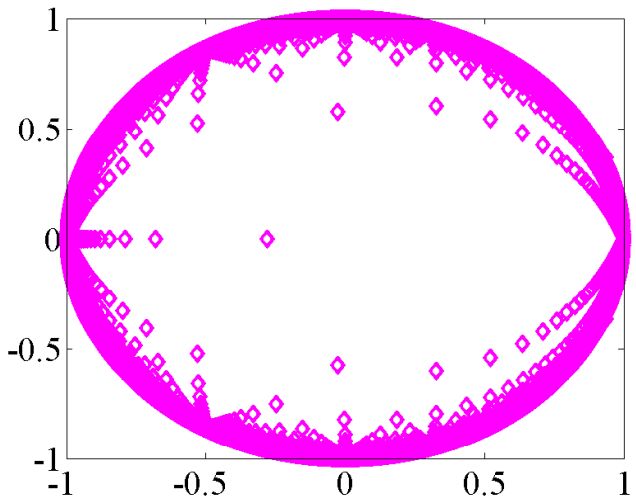

(a)

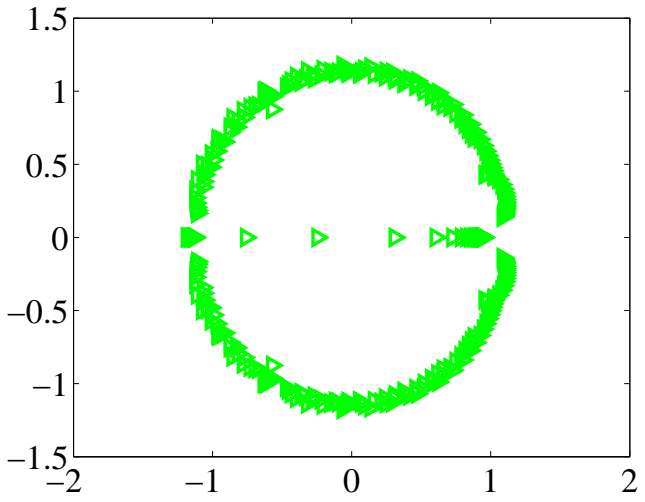

(b)

Figure 5: Numerical simulation showing roots of the polynomial (35) (a) for $m=1000$, within the unit circle indicating the elementary stability of disease free fixed point $\mathcal{E}_{22}$ with $\tau=0.1 \in\left[0, \tau_{0}\right), K=0.282$ (b) for $m=20$, outside/on the unit circle indicating that $\mathcal{E}_{22}$ is unstable fixed point when the delay is increased to $\tau=1.8 \in\left(\tau_{0}, \tau_{1}\right), K=5$ 
fixed point as in the continuous model.

\section{Conclusion}

In this article, the dynamics of the interaction between susceptible and viral phytoplankton with delay as incubation period and consistent numerics are studied. Initially, the model in [11] has been transformed, by replacing the incubation class by a discrete delay to form a system of delay differential equations. A part from confirming the results in [11], this study extend the model in several respects. Furthermore, a suitable numerical scheme, NSFD method, is proposed which approximate and replicate the dynamical properties of the continuous nonlinear delay system. Some of the findings of the study are outlined below:

(i) Three equilibria points are obtained. The trivial and the disease free equilibria are unconditionally inside the biological feasible region, while for the disease free equilibrium, the carrying capacity must be greater than certain threshold value depending on the delay. With this delay and carrying capacity of the susceptible phytoplankton as hopf bifurcation parameters, it is shown that in the absence of viral infection, the disease free equilibrium is both locally and globally asymptotically stable for all delays whenever the carrying capacity is below the threshold value. The ecological implication/interpretation of this, is that, when the carrying capacity of the susceptible phytoplankton population density is less than the threshold value, the viral phytoplankton population will face extinction while the susceptible phytoplankton will grow up to the maximum carrying capacity for any value of the delay. Hence the disease will die out in the phytoplankton population.

(ii) When the carrying capacity is greater than the threshold in (i) above, the endemic equilibrium emerged and is globally asymptotically stable for all delays whenever the carrying capacity is less than another threshold quantity. However, when the carrying capacity is greater than this quantity, the EE is locally asymptotically stable for delay between zero and a critical delay value. Ecologically, it means, the disease will establish itself in the phytoplankton population if the carrying capacity is greater than the threshold value with the initial populations close to the equilibrium. Increasing the delay beyond the critical value, the EE lose its stability and stable periodic solution exists, causing the solution to oscillate around the equilibrium. When the delay reaches the second critical value, EE regains its stability. Consequently, for any fix positive integer, this phenomenon of stability switches from stability to instability and back to stability, eventually, the equilibrium becomes unstable thereafter. 
(iii) A new nonstandard finite difference delay scheme is shown to be dynamically consistent with dynamics of the continuous model. This is the first time a delayedNSFD scheme is constructed for this model and analyzed.

(iv) Numerical simulations are employed to illustrate the analytical results using the NSFD scheme. Furthermore, the effect of delay on the infectivity of phytoplankton has been illustrated.

(v) It is worth noting here that, our model confirms all the results in [11] and beyond. First, in [11], the bifurcation parameter is transfer rate from susceptible to incubation class while here, the bifurcation parameters are delay and carrying capacity. Secondly, global results in the equilibria are established. Lastly, a reliable numerical scheme (NSFD) for the model has been formulated and used to testify the theoretical results and beyond.

\section{Acknowledgments}

The author acknowledges the financial supports of South African DST/NRF SARChI chair on Mathematical Models and Methods in Bioengineering and Biosciences $\left(M^{3} B^{2}\right)$, Department of Mathematics and Applied Mathematics, University of Pretoria and MacArthur Foundation, Bayero University, Kano, Nigeria. The support of the DSTNRF Centre of Excellence in Mathematical and Statistical Sciences (CoE-MaSS) towards this research is hereby acknowledged. Opinions expressed and conclusions arrived at, are those of the author and are not necessarily to be attributed to CoE.

\section{References}

[1] R. Anguelov, J.M.-S. Lubuma, Contribution to the mathematics of the nonstandard finite difference methods and applications, Numer. Meth. Part. Differ. Equ. 17 (2001) 518-543.

[2] R. Anguelov, J.M.-S. Lubuma, Nonstandard finite difference method by nonlocal approximation, Math. Comput. Simul. 61 (2003) 465-475.

[3] R. Anguelov, J.M.-S. Lubuma, M. Shillor, Dynamically consistent nonstandard finite difference schemes for continuous dynamical systems, Discret. Contin. Dyn. Syst. (2009) 34-43.

[4] V.R. Arenas, G. Gonzalez-Parra, A nonstandard dynamically consistent numerical scheme applied to obesity dynamics, J. Appl. Math. (2008).

[5] T.H.B. Christopher, Retarded differential equations, J. Comput. Appl. Math. 125(1) (2000) 309-335. 
[6] A.C. Baudoux, A.A.M. Noordeloos, M.J.W. Veldhuis, C.P.D. Brussaard, Virally induced mortality of phaeocystis globosa during two spring blooms in temperate coastal waters, Aquat. Microb. Ecol. 44 (2006) 207-217.

[7] E. Beretta, Y. Kuang, Geometric stability switch criteria in delay differential systems with delay dependent parameters, SIAM J. Math. Anal. 33(5) (2002) 1144-1165.

[8] C.P.D. Brussaard, R. Thyrhaug, D. Marie, G. Bratbak, Flow cytometric analyses of viral infection in two marine phytoplankton species, Micromonas pusilla (Prasinophyceae) and Phaeocystis pouchetii (Prymnesiophyceae), J. Phycol. 35(5) (1999) 941-948.

[9] K.L. Cooke, P. van den Driessche, On zeros of some transcendental equations, Funkcialaj Ekvacioj 29 (1986) 77-99.

[10] R.V. Culshaw, S. Ruan, A delay-differential equation model of HIV infection of $\mathrm{CD}_{4}^{+} \mathrm{T}$ - cells, Math. Biosci. 165 (2000) 27-39.

[11] J. Dhar, A.K. Sharma, The role of viral infection in phytoplankton dynamics with the inclusion of incubation class, Nonlinear Anal. Hybrid Syst. 4 (2010) 9-15.

[12] O. Diekmann, J.A.P. Heesterbeek, Mathematical epidemiology of infectious diseases, Model building analysis and interpretation, Wiley, New York, 2000.

[13] Y. Dumont, J.M. Tchuenche, Mathematical studies on the sterile insect technique for the Chikungunya disease and Aedes albopictus, J. Math. Biol. 65 (2012) 809854 .

[14] Y. Eissler, R.A. Quiñones, The effect of viral concentrate addition on the respiration rate of Chaetoceros gracilis cultures and microplankton from a shallow bay (Coliumo, Chile), J. Plankton Res. 25(8) (2003) 927-938.

[15] K. Engelborghs, T. Luzyanina, D. Roose, Numerical bifurcation analysis of delay differential equations, J. Comput. Appl. Math. 125(1) (2000) 265-275.

[16] S.M. Garba, A.B. Gumel, A.S. Hassan, J.M.-S. Lubuma, Switching from exact scheme to nonstandard finite difference scheme for linear delay differential equation, Appl. Math. Comput. 258 (2015) 388-403.

[17] S.M. Garba, A.B. Gumel, J.M.-S. Lubuma, Dynamically-consistent non-standard finite difference method for an epidemic model, Math. Comput. Model. 53 (2011) $131-150$. 
[18] S.M. Garba, M.A. Safi, Mathematical analysis of West Nile virus model with discrete delays, ActaMath. Sci . Ser. B 33 (5) (2013) 1439-1462.

[19] S. Goldberg Introduction to Difference Equations, John Wiley and Sons, USA, 1958.

[20] T.H. Gronwall, Note on the derivatives with respect to a parameter of the solutions of a system of differential equations, Anal. Math. (1919) 292-296.

[21] J.K. Hale, S.M.V. Lunel, Introduction to functional differential equations, Springer-Verlag, Berlin, Heidelrberg, New York, 1993.

[22] H.W. Hethcote, The mathematics of infectious diseases, SIAM Rev. 42 (2000) 599-653.

[23] E.I. Jury, Theory and applications of the $z$-transform method, Robert E. Krieger publishing Co., Huntington, New York, 1964.

[24] J. P. LaSalle, The Stability of Dynamical Systems, Regional Conference Series in Applied Mathematics, SIAM., Philadelphia, USA, 1976.

[25] M.Y. Li, H. Shu, Joint effects of mitosis and intracellular delay on viral dynamics: two-parameter bifurcation analysis, J. Math. Biol. 64 (2012) 1005-1020.

[26] M.Y. Li, X. Lin, H. Wang, Global hopf branches and multiple limit cycles in a delayed Lotka-Volterra predator-prey model, Discret. Contin. Dyn. Syst. Ser. B 19 (3) (2014) 747-760.

[27] T. Luzyanina, D. Roose, Numerical stability analysis and computation of hopf bifurcation points for delay differential equations, J. Comput. Appl. Math. 72(2) (1996) 379-392.

[28] R.E. Mickens, Nonstandard finite difference models of differential equations, World scientific, 1994.

[29] R.E. Mickens, Calculation of denominator functions for nonstandard finite difference schemes for differential equations satisfying a positivity condition, Numer. Methods Partial Differ. Equ. 23 (2007) 672-691.

[30] S.M. Moghadas, M.E. Alexander, B.D. Corbett, A.B. Gumel, A positivity preserving Mickens-type discretization of an epidemic model, J. Differ. Equ. Appl. 9 (11) (2003) 1037-1051.

[31] M. Rehim, M. Imran, Dynamical analysis of a delay model of phytoplanktonzooplankton interaction, Appl. Math. Model. 36 (2012) 638-647. 
[32] J.J.H. Reynolds, J.A. Sherratt, A. White, Stability switches in a host-pathogen model as the lenght of a time delay increases, J. nonlinear sci. (2013)

[33] T. Saha, M. Bandyopadhyay, Dynamical analysis of toxin producing phytoplanktonzooplankton interactions, Nonlinear Anal. Real World Appl. 10 (2009) 314-332.

[34] S.T. Smayda, What is a bloom? A commentary, Limnol. Oceonogr. 42 (1997) 1132-1136.

[35] S. A. Kimmance, P. D. Brussard, Estimation of viral-induced phytoplankton mortality using the modified dilution method, Manual of aquatic viral ecology (2010): 65-73.

[36] Y. Wang, J. Wei, Global dynamics of Cholera model with time delay, Int. J. Biomath. 6(1) (2013) 1250070-1-1250070-18.

[37] C. Xu, Bifurcations for a phytoplankton model with time delay, Electron. J. Differ. Equ. 148 (2011) 1-8. 